\title{
Coiled coils as possible models of protein structure evolution
}

\author{
Zoltán Gáspári ${ }^{1, *}$ and László Nyitray ${ }^{2, *}$ \\ ${ }^{1}$ Institute of Chemistry, Eötvös Loránd University, \\ Pázmány Péter sétány 1/A, 1117 Budapest, Hungary \\ ${ }^{2}$ Department of Biochemistry, Eötvös Loránd University, \\ Pázmány Péter sétány 1/C, 1117 Budapest, Hungary \\ * Corresponding authors \\ e-mail: szpari@chem.elte.hu; nyitray@elte.hu
}

\begin{abstract}
Coiled coils are formed by two or more $\alpha$-helices wrapped around one another. This structural motif often guides di-, tri- or multimerization of proteins involved in diverse biological processes such as membrane fusion, signal transduction and the organization of the cytoskeleton. Although coiled coil motifs seem conceptually simple and their existence was proposed in the early 1950s, the high variability of the motif makes coiled coil prediction from sequence a difficult task. They might be confused with intrinsically disordered sequences and even more with a recently described structural motif, the charged single $\alpha$-helix. By contrast, the versatility of coiled coil structures renders them an ideal candidate for protein (re)design and many novel variants have been successfully created to date. In this paper, we review coiled coils in the light of protein evolution by putting our present understanding of the motif and its variants in the context of structural interconversions. We argue that coiled coils are ideal subjects for studies of subtle and large-scale structural changes because of their well-characterized and versatile nature.
\end{abstract}

Keywords: charged single $\alpha$-helix; coiled coil; intrinsically disordered protein; protein design; protein structure.

\section{Introduction}

The existence of the coiled coil motif was proposed by Francis Crick in 1952 based on crystallographic observations (1). The first atomic level structure was reported in 1981 (2), and a large number of different coiled coils from a wealth of proteins has been discovered since then. The structures currently known represent a wide range of variations of the common coiled coil theme (3-5). The structural information gathered yielded insight into the atomic level details of structure stabilization and organization of this structural unit. There are many excellent reviews detailing coiled coil structure, function and design (3-9).

In this paper, we organize our current understanding of coiled coils from a novel perspective, protein structural evo- lution, a field that is of emerging interest among theoretical and experimental structural biologists $(10,11)$. Our present knowledge has allowed successful rational redesign of existing coiled coil motifs (8). More recently, the possibility of interconverting coiled coils to different structural classes such as amyloid-type aggregates (12), intrinsically disordered segments or charged single $\alpha$-helices (CSAHs) has been raised. In this review, we argue that the abundance, structural and functional versatility, as well as the existence of welldescribed stabilizing interactions render coiled coils ideal models for investigating structural changes in proteins that might occur during evolution, ranging from changes in stability through alterations in oligomeric state and/or topology to fold transitions. It must be stressed that (re)constructing possible evolutionary pathways differs from classical design studies as all proposed alterations should be reasonably accessible by mutations and all stages should retain some functionality or at least be harmless within the organism. We also note that the possibility that mutations in coiled coils can lead to structural changes does not necessarily mean that such changes played a major role or indeed occurred during evolution. Nevertheless, this neither prevents their use in model studies nor excludes the interpretation of existing data from this point of view.

\section{The classical coiled coil structure}

Coiled coils are unique among protein structural motifs in the sense that they can readily be described by means of relatively simple parametric equations (4) (Figure 1). These equations, originally compiled by Crick (13) and adopted for real structures later $(14,15)$, describe the positions of backbone atoms along the two or more helices wrapped around each other resulting in the coiled coil. At first sight this might suggest that the coiled coil motif is always well-defined, rather rigid and does not change easily in the course of their function and molecular evolution. By contrast, as we will point out below, our present knowledge shows rather the opposite of this notion.

The classical picture of a coiled coil consists of two righthanded parallel $\alpha$-helices wrapped around each other in a left-handed supercoil. The typical helix crossing angle for such an arrangement is around $22^{\circ}$ and the pitch is approximately $140 \AA$ (16). As $\alpha$-helices have around 3.6 residues per turn, the supercoiling is needed to ensure that the interhelical interface is formed by side chains on average 3.5 residues apart. Indeed, in classical coiled coils, every third and then fourth residue is hydrophobic, resulting in the classic 'heptad' repeat pattern abcdefg, where the positions $d$ and 


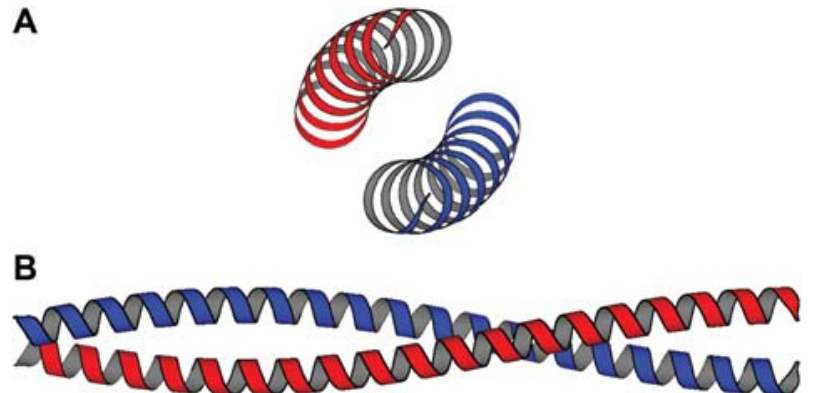

Figure 1 Ribbon representations of an idealized coiled coil computed using the principles in Crick's equations as described in Ref. (13).

(A) View of a 28-residue coiled coil down the supercoil axis. (B) Side view of a long segment of 1.5 superhelical turns. Figures drawn with MOLSCRIPT (105).

$a$ form the interaction layers characteristic of coiled coils. The packing is often referred to as knobs-into-holes meaning that a side chain from one of the helices is packed between four side chains of the other. In two-stranded coiled coils, one of the residues forming the hole is also itself a knob and vice versa. The geometry of the side chains involved can be perpendicular, parallel or between these two extremes, acute, as defined by the relative orientation of the $C \alpha-C \beta$ vectors of the residue in question and the helix face of the opposite chain (8). The $a$ and $d$ layers show characteristic differences in this regard (Figure 2). Beyond the role of hydrophobic side chains at the core $a$ and $d$ positions, coiled coils can use intra- and interhelical electrostatic interactions to tune their stability, especially those between the flanking $e$ and $g$ positions of neighboring chains. It is notable that knobs-intoholes packing are not restricted to coiled coils; it has been observed in local $\alpha$-helix interactions such as between the roughly perpendicular packing of $\alpha$-helices from the regu- latory light chain and the IQ heavy chain in conventional myosins (17).

A typical example of a regular classical two-stranded coiled coil is the leucine zipper motif found in several transcription regulator proteins. The largely regular structure of classical coiled coils suggests that they can be optimal targets of protein design studies - indeed, as will be detailed below, coiled coil design has been recognized as the most successful target of protein engineering (8).

\section{Structural variations (coiled coil topologies)}

In addition to the classical, two-stranded parallel coiled coils described above, many other forms have been described to date, as demonstrated by the recently compiled periodic table of coiled coils (18). An important common aspect is that all of them exhibit knobs-into-holes type interactions to some extent. It should be noted that the precise distinction between coiled coils and other structures is not always straightforward (4). However, owing to the quasi-continuous nature of protein conformations, this is true for many other structural motifs in proteins (19).

In general, structures recognized as coiled coils currently represent a wide range of superhelical arrangements that can be classified according to the number and relative direction of constituent helices as well as the handedness of the supercoil. Currently, the highest-order coiled coil considered, with a single hydrophobic core, is a hexamer, whereas even larger circular structures (dodecamer is the largest) can arise from the bending of a complex structure of pairwisely interacting helices (18). A parallel seven-stranded coiled coil was described in an engineered GCN4 Leu-zipper in which all $e$ and $g$ positions were replaced by Ala residues. High resolution structure of this heptamer revealed a large tubular channel with an unusual heptad register shift between adjacent staggered helices (20). An $\alpha$-barrel, a 12 chain anti-

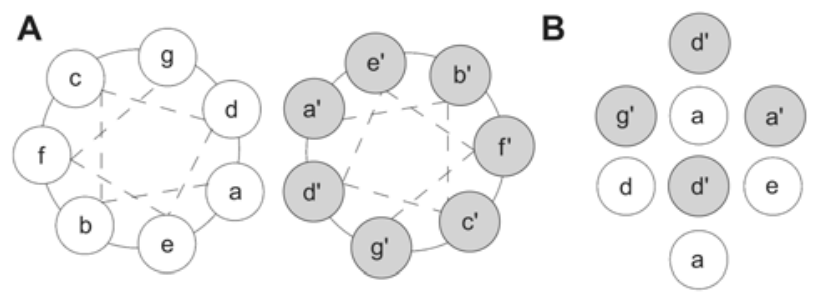

C
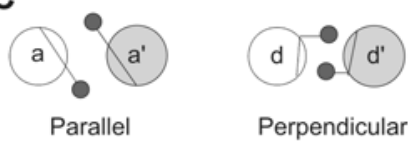

Perpendicular

Figure 2 Schematic representation of a two-stranded canonical coiled coil.

(A) Helical wheel diagram showing the arrangement of residues along the superhelical axis with a straightened interhelical interface to emphasize the heptad repeat structure. (B) Knobs-into-holes packing of the side chains in the $a$ and $d$ positions. The $a$ and $d$ side chains in the middle are knobs fitting into a hole defined by four residues on the other helix (residues with the same notation are one heptad apart). At the same time, they also participate in hole formation for knobs on the interacting chain. (C) Organization of interaction layers in the hydrophobic core of coiled coils. 
parallel coiled coil exists in TolC, an Escherichia coli outer membrane efflux pump (21). The direction of the helices (parallel vs. antiparallel) is also of considerable variation.

In addition to the well-characterized heptad repeat, there are several different repeat sequences, most prominently composed of hendecad units, promoting the formation of coiled coils. Regarding the heptad repeats, there are three common types of its perturbations or discontinuities recognized: skips, stammers and stutters. Skips correspond to the insertion of one residue, stammers to three and stutters to four residues (4). The latter two can be accommodated into the coiled coil without seriously distorting local helical structure. These irregular positions are often highly conserved; skip residues are present in the long coiled coil of myosin tails that are thought to modify the superhelical pitch and affect both the filament structure and regulation through interactions with the head domains $(22,23)$.

The relationships between sequence periodicity, residues per helical turn in the constituent helices and supercoil geometry are well understood $(4,6)$ (Figure 3 ). The positioning of the interacting side chains in the repeated sequences might result in left-handed, straight and right-handed assemblies. Some of these possibilities have even been recognized in the absence of detailed structural information based on theoretical considerations on how interhelical interfaces can be formed by distorting $\alpha$-helical geometry (24). Moreover, such parameters can vary over the length of the coiled coil according to the underlying amino acid sequence. The naturally occurring four-stranded coiled coil at the C-terminus of the tetrabrachion protein contains both hendecads and 15residue pentadecad repeats giving rise to a slightly and a more markedly right-handed supercoil segment (25). The second coiled coil segment of the intermediate filament protein vimentin, a hendecad parallel $\alpha$-helical bundle is followed by a regular left-handed coiled coil (26). Another recently described example is the trimeric coiled coil of the Yersinia adhesin YadA where a structural transition occurs from a firm right-handed to a left-handed supercoil (27).

In a sense, non-heptad repeat units can be regarded as heptads with discontinuities, e.g., hendecads might be viewed as heptads with a stutter. However, it might be more fruitful to consider coiled coils as structures that can have different underlying repeats in their amino acid sequence.
This concept is useful in interpreting structural features (e.g., interaction layers) of coiled coil segments and also possible evolutionary events, e.g., separating the conversion of one repeat to another and subsequent alterations of the newly arisen repeat.

Somewhat surprisingly in the light of the description of 'classical' coiled coils above, the current view is that the 'default' is the trimeric form of coiled coils, meaning that specific interactions are needed to trigger dimer, tetramer or higher order coiled coil formation $(4,8)$. For example, the GCN4 leucine zipper contains Asn in one of the $a$ positions forming an asymmetric hydrogen-bonded interaction destabilizing higher-order structures (28). Interestingly, extracellular $\alpha$-fibrous proteins mostly are three-stranded (including laminin, tenascin, fibrinogen) compared with the mostly twostranded $\alpha$-fibrous proteins (including myosin, tropomyosin, intermediate filament proteins) found intracellularly. Other proteins containing trimeric coiled coil domains include influenza hemagglutinin, heat shock transcription factors, spectrin, $\alpha$-actinin and dystrophin. Three-stranded coiled coils in several autotransporter adhesins have been recently shown to exhibit unusual stabilizing interactions with an elaborate polar network and ions in the core (29). Nevertheless, dimeric ones are by far the most abundant among coiled coils of known structure (18).

The variability of coiled coils is even more increased by heterologous superhelix formation, where different protein chains associate to form asymmetric multimeric structures. Such segments can be formed by single chains as in antiparallel coiled coils formed by sequentially neighboring helices in seryl-tRNA synthetases (30) or separate chains such as in heterodimeric leucine zipper transcription factors (4). Ionic interactions between the helices are considered a major factor governing pair preferences (31).

\section{Folding, stability, dynamics and mechanical properties of coiled coils}

In line with the underlying sequence diversity, the coiled coil motif exhibits considerable variation in terms of stability and dynamics including interconversion of distinct oligomeric species. Length of natural coiled coils is a crucial factor

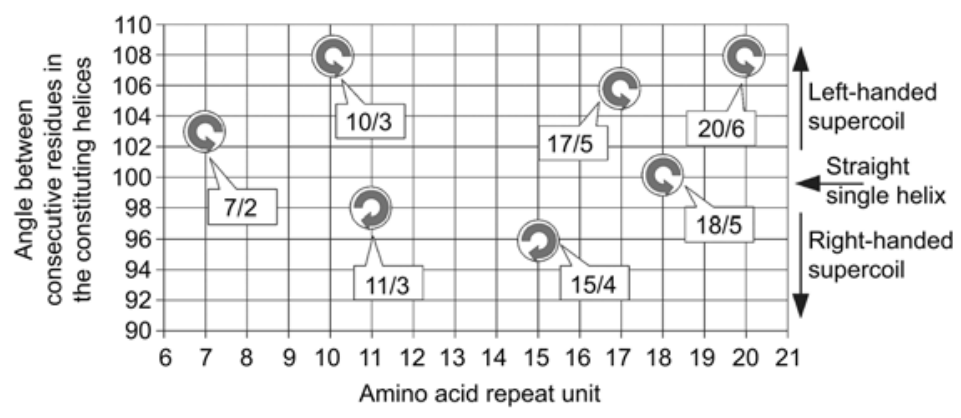

Figure 3 Relationships between repeat sequence, helical and superhelical parameters.

The angle between consecutive residues in the constituent helices is calculated from the residue/turn ratios shown in the call-outs and are defined relative to the supercoil axis. The diagram is based on the data and classification in Ref. (6). 
determining their stability; usually fragments of long coiled coils are not stable, that is their stability is fine-tuned for their biological function $(17,32,33)$.

Coiled coil segments are generally considered as being unfolded in monomeric form and folding upon either supercoil formation or partner recognition and supercoil formation $(34,35)$. The folding/unfolding of coiled coils is generally described by a two-state transition between unfolded monomeric peptide chains and folded coiled coil dimers or oligomers (5). However, more recent studies suggested a more complex folding mechanism $(36,37)$. Several observations suggested the existence of trigger sequences forming stable monomeric $\alpha$-helical structures that are required for the initial interactions that can be propagated to the rest of the structure to form coiled coil structure over even a large distance $(38,39)$. This is in line with studies showing that several regions of long coiled coils can be inherently unstable and require the attachment of a stabilizing extension for structural investigations. In such coiled coils poorly packed residues in the interior of the structure have a propensity for spontaneous unwinding and changes in flexibility $(17,40)$. Recently, 'stability control regions' of tropomyosin were identified that act in synergy over long distances to control coiled coil stability (41). The internal core of coiled coils is also dynamic: based on NMR data, it has been proposed that the asymmetric Asn pair in a leucine zipper is in exchange with an alternate symmetric conformation (42).

Coiled coil formation can also be triggered by environmental changes and such a conformational transition can act as a functional molecular switch (43). The most prominent example is provided by influenza hemagglutinin, where a $\mathrm{pH}$-induced irreversible rearrangement results in coiled coil formation by a segment adopting a loop structure in the native metastable state (44). Another sequence capable of coiled coil formation upon binding to an appropriate partner is the basic DNA-binding part following the leucine zipper region in some transcription factors (45).

An interesting aspect of dynamism is the promiscuity in partner selection sometimes leading to equilibrium of heterogeneous assemblies. The former phenomenon is prevalent in SNARE complex assemblies involved in membrane fusion processes (46), whereas the latter has been observed in several designed coiled coils (8). What is more, a truncated coiled coil segment of scallop myosin was suggested to form antiparallel dimers instead of parallel ones as in the fulllength protein (32). Such a topological heterogeneity was also observed in crystal structures of GCN4 derived peptides that form both parallel and antiparallel tetrameric coiled coils (47).

Coiled coil proteins are valuable models to study mechanical properties of protein structures. It was shown by atomic force microscopy, molecular mechanics simulations and normal mode analysis that the coiled coil of the myosin tail (especially the head-proximal S2 subdomain) is a truly elastic structure that is expected to play an important role in the power stroke of the motor (48-50). Presence of an elastic spring element in processive transport myosins is also important for cargo movement in cells (51). Importance of modular elasticity and flexibility in other coiled coil proteins, such as in tropomyosin, fibrinogen and desmin is also being recognized $(52,53)$.

\section{Coiled coil recognition in protein structures and prediction from sequence}

Coiled coil recognition from three-dimensional structures relies on the identification of knobs-into-holes packing of side chains in neighboring helices, the distinctive hallmark of coiled coils. The program SOCKET has been specifically designed for this task (54) and currently it can be considered as a benchmark in defining coiled coils. However, the cutoff distance used for the definition of interacting residues can be varied to render the algorithm more permissive and several structures considered to be coiled coils by experts can only be recognized with SOCKET when considerably elevated cut-off values are used (18). SOCKET has been used to define a relational coiled coil database (55) and to define data sets of 'known' coiled coils for comparative studies (56).

A somewhat different task is the prediction of coiled coil regions in proteins from their sequence. Despite the apparently simple nature of sequence determinants of coiled coils, the number of algorithms currently available with different underlying concepts (57-67) indicates that recognizing all coiled coils accurately is not trivial. We provide a list of several widely used and some novel methods in Table 1 . The most often used program is COILS (58) which is based on amino acid frequency profiles (implemented as position specific scoring matrices, PSSMs) derived from a set of known coiled coil segments. More recent methods often use Hidden Markov Models (HMMs; Table 1). Although they vary considerably in their underlying concept, practically all of the current methods (except SOSUIcoil, Table 1) are designed to detect uninterrupted heptad repeats $(4,68)$. Another important aspect is that they usually implement a minimum length threshold for coiled coils that is longer than the shortest ones identified in protein structures, which, in turn, can also be recognized differently by experts as there is no consensus on the minimum length of coiled coil structures. These are limitations that should be considered when interpreting coiled coil predictions on full proteomes.

In addition to the presence of a coiled coil segment in a sequence, there are more sophisticated prediction methods to characterize these regions in more detail. The authors of the SOSUIcoil approach have also described and incorporated a method for the detection of fragile points, i.e., regions with less stability in coiled coils (67). They found good agreement of the predictions with electron microscopy and X-ray data.

The program MultiCoil, in principle an extension of PairCoil, was specifically developed to discriminate between two- and three-stranded coiled coils (63). The PrOCoil web server is designed for the same task (http://www.bioinf.jku.at/ software/procoil/). The recently described SpiriCoil approach is able to differentiate between all coiled coil architectures of presently known structure. 
Table 1 List of several coiled coil prediction methods.

\begin{tabular}{|c|c|c|c|}
\hline Name & $\begin{array}{l}\text { Principle of coiled coil } \\
\text { recognition }\end{array}$ & References & URL \\
\hline COILS & PSSM & $(58)$ & http://www.ch.embnet.org/software/COILS_form.html \\
\hline PCOILS & PSSM & (59) & http://toolkit.tuebingen.mpg.de/pcoils \\
\hline $\begin{array}{l}\text { PairCoil, } \\
\text { PairCoil2 }\end{array}$ & $\begin{array}{l}\text { Pairwise residue correlations } \\
\text { within the heptads }\end{array}$ & $(60,61)$ & http://groups.csail.mit.edu/cb/paircoil2/ \\
\hline MARCOIL & HMM & $(62)$ & http://www.isrec.isb-sib.ch/BCF/Delorenzi/Marcoil/index.html \\
\hline MultiCoil & $\begin{array}{l}\text { Pairwise residue correlations } \\
\text { within the heptads }\end{array}$ & $(63)$ & http://groups.csail.mit.edu/cb/multicoil/cgi-bin/multicoil.cgi/cgi-bin/multicoil \\
\hline CCHMM_PROF & HMM & $(64)$ & http://gpcr.biocomp.unibo.it/cgi/predictors/cchmmprof/pred_cchmmprof.cgi \\
\hline SpiriCoil & HMM & $(65)$ & http://supfam.cs.bris.ac.uk/SUPERFAMILY/spiricoil/ \\
\hline Amphiseach & Residue hydrophobicity & $(66)$ & - \\
\hline SOSUIcoil & $\begin{array}{l}\text { Canonical discriminant } \\
\text { analysis }\end{array}$ & $(67)$ & http://bp.nuap.nagoya-u.ac.jp/sosui/coil/submit.html \\
\hline PrOCoil & $\begin{array}{l}\text { (uses input from } \\
\text { other methods and predicts } \\
\text { oligomerization state using } \\
\text { support vector machines) }\end{array}$ & - & http://www.bioinf.jku.at/software/procoil/ \\
\hline
\end{tabular}

CC, coiled coil; CSAH, charged single $\alpha$-helix; DYNLL, LC8 dynein light chain; HMM, hidden Markov model; PSSM, position-specific scoring matrix.

Perhaps the most complicated issue is the prediction of pair preferences in heteromeric coiled coil structures. This was addressed for leucine zipper interactions with a method scoring all pairwise interactions regarded important in partner specificity of dimeric coiled coils (69). The authors concluded that taking into account $d d^{\prime}, d a^{\prime}, a d^{\prime}, g a^{\prime}$ and $d e^{\prime}$ interactions in addition to the more commonly considered $g e^{\prime}$ and $a a^{\prime}$ pairs increases the accuracy of the predictions.

\section{Occurrence of coiled coils in living organisms}

Coiled coils are present in all domains of life indicating the evolutionary success of a simple yet versatile architecture. They are clearly more abundant in eukaryotes suggesting that they play a role in the increasingly complex intracellular processes of eukaryotic cells. They are predicted in $\sim 10 \%$ of all eukaryotic proteins and less than $5 \%$ in prokaryotes. A comprehensive database was collected for long coiled coils (defined as longer than 70 residues in one chain) in 22 organisms (70). The only long coiled coils are SMC proteins (that belong to the ABC type ATPase family and are involved in structural maintenance of chromosomes) that are conserved in all kingdoms. Interestingly, no coiled coil proteins with longer than 250 residues were identified in all but one of the eubacterial genomes. As expected, archaeal genomes contain all size class of coiled coil proteins similar to eukaryotes. In addition to the SMC proteins, additional long coiled coil domains present in prokaryotes include proteins involved in cell growth and morphology. Several of them have been shown to have a cytoskeletal function and some have been proposed to have an IF-like character (71). Shorter coiled coils that are shared by prokaryotes and eukaryotes are translation IF2s and $\mathrm{AAA}^{+}$ATPases. Coiled coil containing motor proteins (myosins, kinesin, dyneins), membrane teth- ering and vesicle transport proteins are specific for eukaryotes only.

Long coiled coil proteins are four-fold more frequent in the animal kingdom and could reflect early events in the divergence of plants and animals. It has been speculated that some of these coiled coils and their interactions can underlie metazoan differentiated cell and tissue structure (72). Coiled coil sequences limited to mammals include keratins, several centrosomal proteins and blood clotting factors to name just a few. A large number of coiled coil domain containing plant proteins have unknown function. Plant-specific coiled coils include protein kinase and actin binding proteins (73).

A very recent genome-wide survey of coiled coil domains, based on known SCOP superfamilies and hidden Markov models (SpiriCoil database), allowed analysis of coiled coil evolution; it is suggested that coiled coils have arisen independently de novo well over a hundred times and coiled coils with almost all oligomeric states were present in the last universal common ancestor of life (65). Wild distribution of coiled coil proteins in living organisms together with their functional diversity underlies the requirement for evolutionary selection of robust but dynamic and highly adaptable coiled coil structure; in fact, they possess these properties.

\section{Functions of coiled coils}

High prevalence of coiled coil domains across the evolutionary tree of life indicates their significance in many key biological processes. The fact that such a simple architecture could participate in several distinct cellular functions suggest that the rules governing the partner selection of the helices are both complex and easily tunable by evolution as new functions could arise either by tinkering with existing structures or the emergence of novel ones not negatively inter- 
fering with those already present. Their most obvious function is to act as a 'molecular Velcro' to hold together polypeptide chains and subcellular structures. They are present in all classes of proteins - fibrous, globular and membrane proteins. Historically, the $\alpha$-fibrous proteins were first recognized as coiled coils. The MTK proteins (myosin, tropomyosin and keratin) are the archetypes of long parallel dimeric coiled coils and their sequences were used to develop the first coiled coil prediction method, COILS (4).

Currently, myriad and still expanding functions can be assigned to coiled coils and they are still expanding. Coiled coils are the most frequent dimerization, multimerization motifs. Long coiled coils have mostly structural and mechanical roles. They form rod-like rigid, but often discontinuous, flexible structures that could assemble into fibers, meshworks and scaffolds. They are ideally suited to form keratinaceous structures, extracellular matrix components (e.g., laminin, fibrinogen), intermediate and several other types of intracellular filaments (e.g., muscle thick filaments, flagellins). The extracellular cartilage oligomeric matrix protein (COMP) is a five-stranded coiled coil that has one of the highest thermal stability of all proteins (74). They can also function as molecular spacers (e.g., in seryl-tRNA synthetase). One of the longest flexible spacer coiled coil domain $(230 \mathrm{~nm})$ was identified in CENP-E kinesin that provides a flexible, motile tether linking kinetochores to dynamic spindle microtubules (75). Coiled coils could act as molecular switches or 'zippers' in hemagglutinin, in the HIV gp120 protein and in other host-pathogen interactions. They could be organizers in subcellular structures such as in the cytoskeleton, microtubule organizing center, nuclear pore complex, chemotaxis machinery and so on. Coiled coil domains together with ATPase or GTPase domains often function in folding and repair; e.g., prefoldin chaperon in protein folding (76), topoisomerases and helicases in DNA remodeling (77, 78). Transcription elongation is regulated by Hexim which has a bipartite flexible coiled coil domain (79). Coiled coil motifs can also homodimerize and heterodimerize membrane receptor and channel proteins (80).

The dynamic nature of many coiled coils would allow them to interact with various partners and participate in multiple complexes. Some coiled coil predicted sequences are not stable enough to dimerize under physiological conditions and they need some chaperonin-like assistance to zipper up; the DYNLL hub protein has been suggested to act like a coiled coil dimerization engine $(81,82)$. By regulating the stability of coiled coil domains, DYNLL could regulate and fine-tune the activity of the binding partner.

The structural simplicity and reversible nature of coiled coil associations render them a promising target for pharmacological interference (successfully exemplified by viral fusion inhibitors) (83). Recently, an interesting biological application, use of coiled coil proteins for therapeutic purposes as drug delivery systems has been put forward; vitamins and the anticancer drug cisplatin have already been shown to bind to the large internal cavities in the pentameric coiled coil domain of COMP and the tetrameric right-handed coiled coil of the Staphylothermus marinus RHCC protein (84).

\section{Variant design}

Although usually not aimed at understanding structural evolution, design studies provide a wealth of information on how amino acid changes affect coiled coil structure and dynamics and thus the results could easily be interpreted within an evolutionary context in a model system. Coiled coils are one of the most successful subjects of protein design and engineering. Analysis of the side chain-side chain interactions in the hydrophobic core of existing structures led to quick recognition of some basic packing rules in these structures (28). Varying Ile and Leu in the $a$ and $d$ positions in the GCN4 leucine zipper led to dimeric (GCN4-p-IL, having Ile in $a$ and Leu in $d$ positions), trimeric (GCN4-p-II) and tetrameric (GCN4-p-LI) forms (28). In agreement with these preferences, the retro version of the GCN4 leucine zipper forms a tetramer having Leu in $a$ and Ile in $d$ positions (85). For the purpose of this review, it is important to note that other designed peptides, such as p-VI, p-VL, p-LV and p-LL exhibited multiple oligomerization states (28).

It is believed that an Asn in leucine zipers is required to make the dimeric form preferred. This is based on the $\mathrm{H}$ bonding interaction formed between two Asn residues in $a$ positions (consider also the dynamic nature of this interaction mentioned above). Moreover, steric factors also govern multimeric state as both bulky hydrophobic and large hydrophilic side chains prefer dimer formation as they might not easily accommodate the larger interiors in larger assemblies. The designed peptide coil-Ser forms a trimer with two parallel strands, which was in part rationalized on the basis of the presence of a Trp residue in the first $a$ positions which cannot fit into a single layer in a trimeric core (86). A computational approach to distinguish between dimeric and trimeric coiled coils was also developed in the mid-1990s (8) based on amino acid preferences observed in known dimeric and trimeric structures. Somewhat less is known about the role of ionic interactions in coiled coils. However, there are successful designs where partner selection could be directed by engineering salt bridges favorable only in the desired structure. Rational engineering of the GCN4-p-LI peptide resulted in a heterotetrameric structure where the two constituent strands did not form coiled coils in the absence of the other one (87). Experience with such systems suggests that avoiding unfavored interactions is more important than the formation of favored ones (8). Coiled coil peptides have also been designed to form fibers (both amyloid and non-amyloid) upon external triggering by $\mathrm{pH}$ or temperature, or simply upon mixing (88).

\section{Relation of coiled coils to other structural motifs}

Although coiled coils have distinct characteristics making them a unique conformational state, they share similarities with other types of protein structural elements. Instances of knobs-into-holes packing has been observed in several interhelical interactions previously not considered as coiled coils 
(89). The distinguishing feature of coiled coils in this regard is the occurrence of full layers with this interaction in a consecutive manner along the helices. This restriction still enables the recognition of several transmembrane helical interactions as coiled coils in the photosynthetic reaction center by the program SOCKET, although not consistently between the same pairs of homologous helices in the different structures examined (54). This means that this type of interaction is not a key feature in these transmembrane segments.

Coiled coils are often viewed as a specific set of intrinsically disordered proteins (IDPs), defined as having no welldefined structure in their physiological state (90). Coiled coils meet this criterion in the sense that they are unfolded as monomers as specifically observed for several designed heteromeric structures (see above). Moreover, coiled coil sequences have been noted to be predicted to be disordered by IDP prediction algorithms (91). A prominent example of mispredictions is provided by the trimeric coiled coils of autotransporter proteins, having an unusual heptad repeat with asparagine in the $d$ position (29). In general, cross-predictions have been found common in a systematic survey involving several coiled coil and IDP prediction algorithms (56). This can be rationalized on the basis of similar amino acid composition of coiled coils and IDPs, the most striking differences being in the abundance of Leu, Pro and Gly (Figure 4).

The recently identified CSAH structural motif $(92,93)$ also often overlaps with predicted coiled coil segments. More importantly, in several proteins CSAHs are localized in positions of coiled coils in homologous sequences (93), indicating that they might have been interconverted during evolution. The notion that coiled coils can be converted to distinct structural classes is reinforced by the designed peptide $\operatorname{cc} \beta$, which forms amyloid-type aggregates at elevated temperatures (12). This is in accordance with some observations showing that amyloid formation is an inherent property of polypeptide chains $(94,95)$.

Coiled coils have also been used as initial templates to design model transmembrane helices, although it must be noted that the resulting sequences were far from the templates used and the exact mode of interaction of the helices within the membranes could not be reliably identified (96, 97).

\section{Coiled coils and protein structural evolution}

Protein structural evolution is a process different from rational protein engineering mainly because evolution does only allow specific types of sequence alteration constrained by the need of a functional or at least harmless variant of the protein in question. Thus, reconstructing evolutionary events or testing possible routes should take this consideration into account, as is done in studies resurrecting ancient proteins (98).

Detection of evolutionary relationships between proteins relies on comparisons of their sequence and structure. Coiled coils represent a special case in this regard as their relatively simple repeating sequence suggests that such structures might have emerged multiple times during protein evolution, meaning that not all present coiled coils are homologous. Even similar coiled coils might be the result of convergent evolution. Indeed, recent investigations of protein superfamilies suggest the emergence of coiled coils over 100 times independently (65). This is a feature most probably common with other low complexity protein segments such as IDPs and is consistent with the increased abundance of coiled coil proteins in archaea and eukaryotes (also having an excess of IDPs relative to bacteria) again justified by phylogenetic analysis (65). By contrast, the observations derived from design studies suggest that some coiled coils with different topologies might be more closely related than expected based on strict sequence comparisons. To further complicate the issue, finding different structural segments in the place of coiled coils in homologous proteins does not necessarily mean that the two segments are related as they could have arisen by independent insertion-deletion events not necessarily detectable even at the DNA level. Nevertheless, even if it might not be always feasible to reconstruct the exact

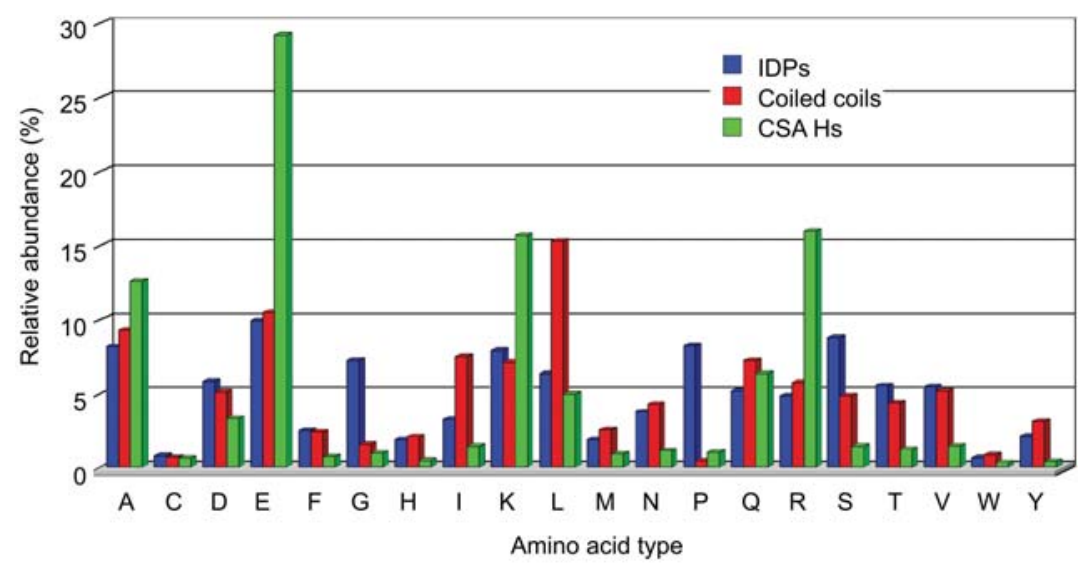

Figure 4 Amino acid abundance in IDPs, coiled coils and CSAHs.

IDP sequences were taken from DisProt, coiled coils from a database generated by running SOCKET on structures listed in the PDB SELECT database, CSAHs from segments identified in Ref. (92). Data taken from Ref. (56). 
evolutionary history of a coiled coil, investigating hypotheses on its possible structural transitions can still be of scientific value.

In addition to point mutations and small insertions altering a few amino acids, the repetitive nature of coiled coil sequences could also allow repeat expansion/contraction events affecting larger segments. There are several other types of helical proteins composed of repeated units with variations (99), but coiled coils differ from these because of having a relatively short repeat unit, the heptad/hendecad (although any multiples of these could serve as a 'unit' for expansion) and that addition of units would elongate the constituent helices rather than inserting a new, more or less independent structural module. Variations in coiled coil sequences might result in superhelical structures differing in length, geometry, partner specificity and/or dynamics from the parent molecule. Insertions might result in distortion of the repeat pattern by inserting a skip, a stutter or a stammer, all of which are compatible with coiled coil formation but alter the local geometry of the superhelix. Mutation of the core residues could affect stability, oligomeric state and together with the alteration of ionic interactions might alter partner selection and strand orientation. Based on the analysis of natural variants, it has been suggested that there is a delicate balance governing coiled coil geometry and stability between the deviation from the optimal helical geometry and helix-helix packing interactions (4). Thus, it is expected that there are relatively short paths of small evolutionary changes that interconvert coiled coils with different topologies while keeping superhelical geometry at each step. Such paths might proceed through states where mixtures of different forms and/or relatively unstable structures exist. These nevertheless might be functional if one of the forms is capable of fulfilling a task beneficial for the organism. Dynamically interconverting ensembles are a manifestation of intermolecular dynamism, an extension of intramolecular flexibility that is considered a key factor in protein structural evolution (11). Coiled coils might also be formed conditionally, e.g., upon $\mathrm{pH}$ change just like in influenza hemagglutinin and HIV gp120, and such transient or conditional structures might be stabilized or destabilized to yield new variants. Discrepancies in coiled coil prediction might reflect different propensities of protein segments to form coiled coils (Figure 5A). Intrinsic disorder of monomers of coiled coils is an important property for molecular recognition; the coiled coil propensity together with considerable structural plasticity could be highly advantageous to evolve novel hetero coiled coils and multichain coiled coil complexes.

Coiled coil-IDP interconversion might proceed through different courses. Leu and Pro, two of the residues exhibiting large differences in their abundance in these two structural classes, are encoded by neighboring boxes in the standard genetic code. Thus, these residues can be easily interconverted by point mutations and several such mutations might be sufficient to convert coiled coils to IDPs or in reverse. Although this would be an attractive 'direct route' between the two types of structures, segments predicted to be both coiled coils and IDPs have an amino acid distribution resembling those of CSAHs, although not always recognized as such by the current algorithms. This suggests that an indirect route might exist proceeding through the CSAH stage. CSAHs are indeed similar, in several aspects, to coiled coils and, in

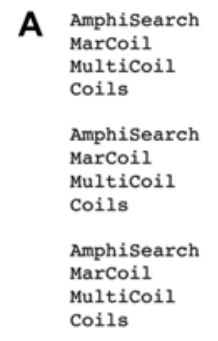

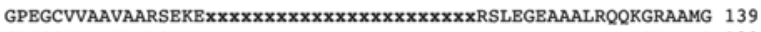

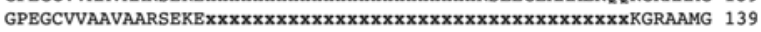
GPEGCVVAAVAARSEKEQLQALNDRFAGYIDKVRQLEAHNRSLEGEAAALRQQKGRAAMG 139

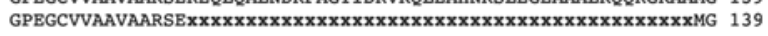

ELYEREVREMRGAVLRLGAARGQLRLEQEHLLEDIAHVRQRLDEEARQREEAEAAARALA 199

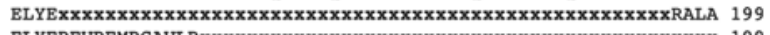

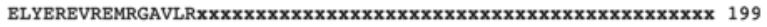

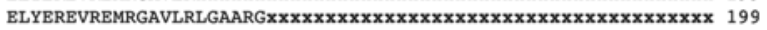

FAQEAEAARVELQKKAQALQEECGYLRRHHQEEVGELLGQI 240

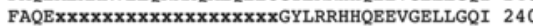
$\begin{array}{ll}F A Q E x x x x x x x x x x x x x x x x x x G Y L R H H Q E E V G E L L Q I & 240 \\ x x x x x x x x x x x x x x x x x x x x x x x x x x x x x x x x E V G E L L G Q I & 240\end{array}$ $\begin{array}{lll}X X X X X X X X X X X X X X X X X X X X X X & \end{array}$

\section{B}

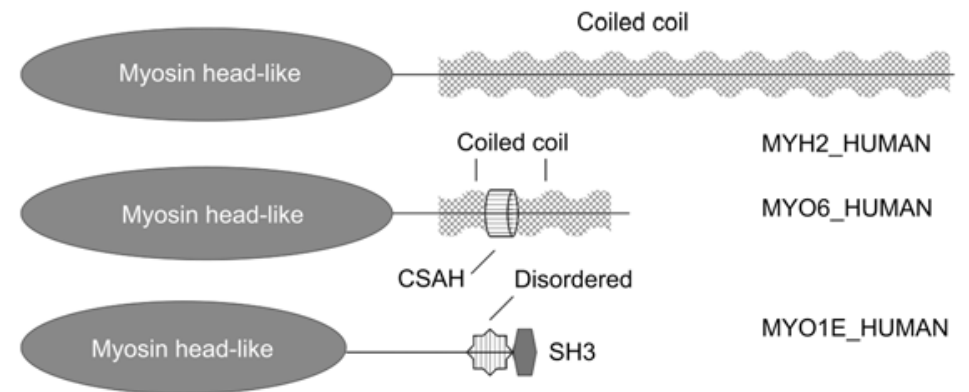

Figure 5 Variations for coiled coil recognition and occurrence in proteins.

(A) Results of coiled coil predictions with different algorithms on the same sequence. A segment of the mouse neurofilament heavy peptide (SwissProt ID: NFH_MOUSE) is shown as an example, residues predicted from coiled coils are masked by ' $\mathbf{x}$ '. Prediction results are taken from Ref. (56). (B) Domain composition of three human myosins. Assignments are based on SwissProt annotations plus CSAH detection (93) as well as IUPred (106). Note that analogous position of segments does not necessarily imply homology. 
other ones to IDPs, and it is highly probable that presentday CSAHs in several proteins such as myosin VI and $\mathrm{X}$ stem from coiled coil segments found in their place in several other myosin families (Figure 5B) $(100,101)$. The fact that some orthologs of certain CSAHs or coiled coil domains, even within the vertebrate clan are interchangeable, support the above view (93). Regarding the structural evolution of the coiled coil motifs it would also be possible that they evolved from the rearrangement of existing $\alpha$-helices in proteins; however, the recently presented SpiriCoil analysis of coiled coil structural domains across all sequenced genomes argues against this hypothesis (65).

The possibility of coiled coils to change their oligomerization state during evolution looks plausible in the light of numerous design studies showing the feasibility of this with just a few amino acid changes. By contrast, data in the recently compiled SpiriCoil database show that coiled coils have essentially not undergone such alterations except the assembly of two two-stranded coiled coils to form a fourhanded superhelix (65). However, the observation that some CSAHs might have emerged from coiled coils can be regarded as an example of more recent changes in the oligomerization state (102).

Last but not least, it is important to stress that using coiled coils as models to understand aspects of protein structural evolution does not mean that the changes that might be observed had indeed occurred during evolution. Proteins exist in an environment within organisms where a multitude of selection constraints are in operation that might preclude structural transitions that can be easily modeled experimentally. Actual evolutionary routes from one state to the other might be highly complex and require detailed phylogenetic studies to get uncovered. Thus, our proposed network of interconverting helical structures (Figure 6) might be plausible during protein evolution but it might well be that some of the paths have been never taken by natural sequences.

\section{Expert opinion and outlook}

Despite the relative simplicity of their sequences, coiled coils constitute an extremely versatile class of proteins both in terms of structure and function. They provide one of the bestunderstood examples of the links between sequence and structural features, knowledge which has been extensively used in successful protein design studies. This growing knowledge allows the design of structural transitions between coiled coils with various topologies and even also leading to other structures with step-by-step alterations keeping some functionality at each step. The recent discovery of a catalytic site within a coiled coil motif (103) and observations that poorly structured proteins can act as enzymes (104) provide the possibility of devising and performing functional assays to explore such evolutionary scenarios. We believe that along with recent suggestions (11), protein dynamics - both internal motions and dynamic association with different partners - is one of the key factors governing structural and functional transitions. Thus, dynamic aspects

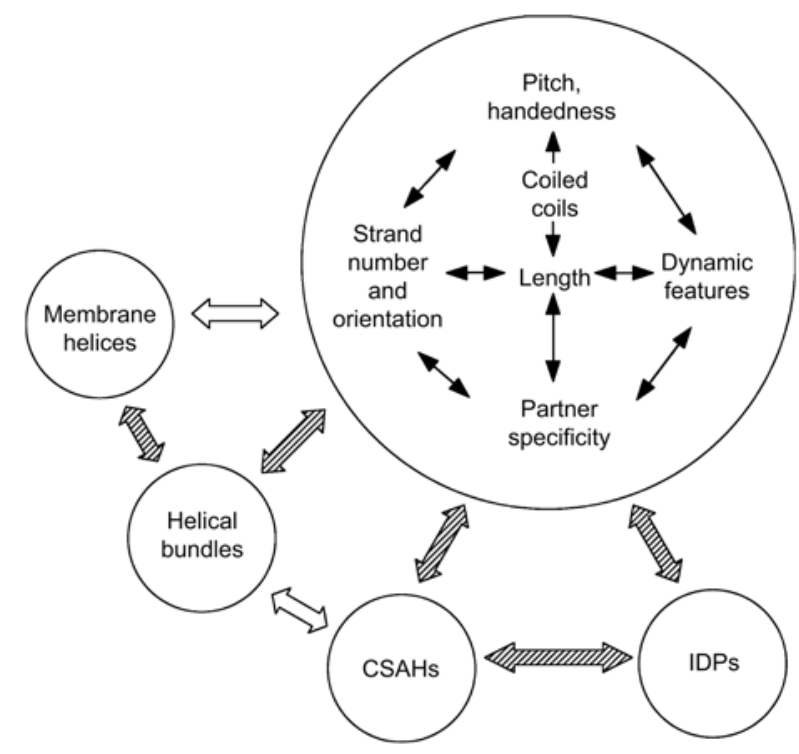

Figure 6 A possible depiction of suggested interrelationships of coiled coil features as well as coiled coils and other structural motifs in the course of protein structural evolution.

Routes that might be less prevalent are shown with hollow arrows.

of proteins, including coiled coils, should be considered in the design and considered in the evaluation of the variants investigated.

We expect that our understanding of the relationships of protein sequence, structure, dynamics and function will be facilitated by studies modeling possible evolutionary transitions in proteins. For such investigations, coiled coils constitute well-characterized and ready-to-use systems of varying complexity, rendering them ideal targets.

\section{Acknowledgements}

This work was supported by grants from the Hungarian Scientific Research Fund (OTKA F68079, K72973, K61784 and NI68466) and ICGEB (CRP/HUN09-03). The European Union and the European Social Fund have provided financial support to the project under the grant agreement no. TÁMOP 4.2.1./B-09/1/KMR-20100003. Z.G. also acknowledges a János Bolyai Research Fellowship.

\section{References}

1. Crick FH. Is $\alpha$-keratin a coiled coil? Nature 1952; 170: 882-3.

2. Wilson IA, Skehel JJ, Wiley DC. Structure of the hemagglutinin membrane glycoprotein of influenza virus at $3 \AA$ resolution. Nature 1981; 289: 366-73.

3. Burkhard P, Stetefeld J, Strelkov SV. Coiled coils: a highly versatile protein folding motif. Trends Cell Biol 2001; 11: 82-8.

4. Lupas AN, Gruber M. The structure of $\alpha$-helical coiled coils. Adv Protein Chem 2005; 70: 37-78. 
5. Mason JM, Arndt KM. Coiled coil domains: stability, specificity, and biological implications. ChemBioChem 2004; 5: 170-6.

6. Gruber M, Lupas AN. Historical review: another 50th anniversary - new periodicities in coiled coils. Trends Biochem Sci 2003; 28: 679-85.

7. Rose A, Meier I. Scaffolds, levers, rods and springs: diverse cellular functions of long coiled-coil proteins. Cell Mol Life Sci 2004; 61: 1996-2009.

8 . Woolfson DN. The design of coiled-coil structures and assemblies. Adv Protein Chem 2005; 70: 79-112.

9. Parry DA, Fraser RD, Squire JM. Fifty years of coiled-coils and $\alpha$-helical bundles: a close relationship between sequence and structure. J Struct Biol 2008; 163: 258-69.

10. Pal C, Papp B, Lercher MJ. An integrated view of protein evolution. Nat Rev Genet 2006; 7: 337-48.

11. Tokuriki N, Tawfik DS. Protein dynamism and evolvability. Science 2009; 324: 203-7.

12. Kammerer RA, Kostrewa D, Zurdo J, Detken A, Garcia-Echeverria C, Green JD, Muller SA, Meier BH, Winkler FK, Dobson CM, Steinmetz MO. Exploring amyloid formation by a de novo design. Proc Natl Acad Sci USA 2004; 101: 4435-40.

13. Crick FH. The Fourier transform of a coiled-coil. Acta Crystallogr 1953; 6: 685-9.

14. Offer G, Hicks MR, Woolfson DN. Generalized Crick equations for modeling noncanonical coiled coils. J Struct Biol 2002; 137: 41-53.

15. Offer G, Sessions R. Computer modelling of the alpha-helical coiled coil: packing of side-chains in the inner core. J Mol Biol 1995; 249: 967-87.

16. Seo J, Cohen C. Pitch diversity in $\alpha$-helical coiled coils. Proteins 1993; 15: 223-34.

17. Brown JH, Yang Y, Reshetnikova L, Gourinath S, Suveges D, Kardos J, Hobor F, Reutzel R, Nyitray L, Cohen C. An unstable head-rod junction may promote folding into the compact off-state conformation of regulated myosins. J Mol Biol 2008; 375: 1434-43.

18. Moutevelis E, Woolfson DN. A periodic table of coiled-coil protein structures. J Mol Biol 2009; 385: 726-32.

19. Andersen CAF, Rost B. Secondary structure assignment. In: Bourne PE, Weissig H, editors. Structural bioinformatics. Chichester: John Wiley and Sons, 2003: 341-6.

20. Liu J, Zheng Q, Deng Y, Cheng CS, Kallenbach NR, Lu M. A seven-helix coiled coil. Proc Natl Acad Sci USA 2006; 103: 15457-62.

21. Koronakis V. TolC - the bacterial exit duct for proteins and drugs. FEBS Lett 2003; 555: 66-71.

22. Lowey S, Trybus KM. Common structural motifs for the regulation of divergent class II myosins. J Biol Chem 2010; 285: 16403-7.

23. Offer G. Skip residues correlate with bends in the myosin tail. J Mol Biol 1990; 216: 213-8.

24. Pauling L, Corey RB. Compound helical configurations of polypeptide chains: structure of proteins of the $\alpha$-keratin type. Nature 1953; 171: 59-61.

25. Stetefeld J, Jenny M, Schulthess T, Landwehr R, Engel J, Kammerer RA. Crystal structure of a naturally occurring parallel right-handed coiled coil tetramer. Nat Struct Biol 2000; 7: $772-6$.

26. Nicolet S, Herrmann H, Aebi U, Strelkov SV. Atomic structure of vimentin coil 2. J Struct Biol 2010; 170: 369-76.

27. Alvarez BH, Gruber M, Ursinus A, Dunin-Horkawicz S, Lupas AN, Zeth K. A transition from strong right-handed to canonical left-handed supercoiling in a conserved coiled-coil segment of trimeric autotransporter adhesins. J Struct Biol 2010; 170: 236-45.

28. Harbury PB, Zhang T, Kim PS, Alber T. A switch between two-, three-, and four-stranded coiled coils in GCN4 leucine zipper mutants. Science 1993; 262: 1401-7.

29. Hartmann MD, Ridderbusch O, Zeth K, Albrecht R, Testa O, Woolfson DN, Sauer G, Dunin-Horkawitz S, Lupas AN, Hernandez Alvarez B. A coiled-coil motif that sequesters ions to the hydrophobic core. Proc Natl Acad Sci USA 2009; 106: 16950-5.

30. Cusack S, Berthet-Colominas C, Hartlein M, Nassar N, Leberman R. A second class of synthetase structure revealed by Xray analysis of Escherichia coli seryl-tRNA synthetase at 2.5 Å. Nature 1990; 347: 249-55.

31. Beck K, Dixon TW, Engel J, Parry DA. Ionic interactions in the coiled-coil domain of laminin determine the specificity of chain assembly. J Mol Biol 1993; 231: 311-23.

32. Malnasi-Csizmadia A, Shimony E, Hegyi G, Szent-Gyorgyi AG, Nyitray L. Dimerization of the head-rod junction of scallop myosin. Biochem Biophys Res Commun 1998; 252: 595-601.

33. Trybus KM, Freyzon Y, Faust LZ, Sweeney HL. Spare the rod, spoil the regulation: necessity for a myosin rod. Proc Natl Acad Sci USA 1997; 94: 48-52.

34. Wendt H, Leder L, Harma H, Jelesarov I, Baici A, Bosshard HR. Very rapid, ionic strength-dependent association and folding of a heterodimeric leucine zipper. Biochemistry 1997; 36: 204-13.

35. Zitzewitz JA, Bilsel O, Luo J, Jones BE, Matthews CR. Probing the folding mechanism of a leucine zipper peptide by stopped-flow circular dichroism spectroscopy. Biochemistry 1995; 34: 12812-9.

36. Balakrishnan G, Hu Y, Case MA, Spiro TG. Microsecond melting of a folding intermediate in a coiled-coil peptide, monitored by T-jump/UV Raman spectroscopy. J Phys Chem B 2006; 110: 19877-83.

37. Dragan AI, Privalov PL. Unfolding of a leucine zipper is not a simple two-state transition. J Mol Biol 2002; 321: 891-908.

38. Steinmetz MO, Jelesarov I, Matousek WM, Honnappa S, Jahnke W, Missimer JH, Frank S, Alexandrescu AT, Kammerer RA. Molecular basis of coiled-coil formation. Proc Natl Acad Sci USA 2007; 104: 7062-7.

39. Steinmetz MO, Stock A, Schulthess T, Landwehr R, Lustig A, Faix J, Gerisch G, Aebi U, Kammerer RA. A distinct 14 residue site triggers coiled-coil formation in cortexillin I. EMBO J 1998; 17: 1883-91.

40. Li Y, Brown JH, Reshetnikova L, Blazsek A, Farkas L, Nyitray L, Cohen C. Visualization of an unstable coiled coil from the scallop myosin rod. Nature 2003; 424: 341-5.

41. Hodges RS, Mills J, McReynolds S, Kirwan JP, Tripet B, Osguthorpe D. Identification of a unique 'stability control region' that controls protein stability of tropomyosin: a twostranded $\alpha$-helical coiled-coil. J Mol Biol 2009; 392: 747-62.

42. Junius FK, Mackay JP, Bubb WA, Jensen SA, Weiss AS, King GF. Nuclear magnetic resonance characterization of the Jun leucine zipper domain: unusual properties of coiled-coil interfacial polar residues. Biochemistry 1995; 34: 6164-74.

43. Oas TG, Endow SA. Springs and hinges: dynamic coiled coils and discontinuities. Trends Biochem Sci 1994; 19: 51-4.

44. Carr CM, Chaudhry C, Kim PS. Influenza hemagglutinin is spring-loaded by a metastable native conformation. Proc Natl Acad Sci USA 1997; 94: 14306-13. 
45. Krylov D, Olive M, Vinson C. Extending dimerization interfaces: the bZIP basic region can form a coiled coil. EMBO J 1995; 14: 5329-37.

46. Pfeffer SR. Unsolved mysteries in membrane traffic. Annu Rev Biochem 2007; 76: 629-45.

47. Yadav MK, Leman LJ, Price DJ, Brooks CL III, Stout CD, Ghadiri MR. Coiled coils at the edge of configurational heterogeneity. Structural analyses of parallel and antiparallel homotetrameric coiled coils reveal configurational sensitivity to a single solvent-exposed amino acid substitution. Biochemistry $2006 ; 45$ : 4463-73.

48. Adamovic I, Mijailovich SM, Karplus M. The elastic properties of the structurally characterized myosin II S2 subdomain: a molecular dynamics and normal mode analysis. Biophys J 2008; 94: 3779-89.

49. Root DD, Yadavalli VK, Forbes JG, Wang K. Coiled-coil nanomechanics and uncoiling and unfolding of the superhelix and $\alpha$-helices of myosin. Biophys J 2006; 90: 2852-66.

50. Schwaiger I, Sattler C, Hostetter DR, Rief M. The myosin coiled-coil is a truly elastic protein structure. Nat Mater 2002; 1: 232-5.

51. Nagy A, Piszczek G, Sellers JR. Extensibility of the extended tail domain of processive and nonprocessive myosin $\mathrm{V}$ molecules. Biophys J 2009; 97: 3123-31.

52. Kiss B, Karsai A, Kellermayer MS. Nanomechanical properties of desmin intermediate filaments. J Struct Biol 2006; 155: 327-39.

53. Lakkaraju SK, Hwang W. Modulation of elasticity in functionally distinct domains of the tropomyosin coiled-coil. Cell Mol Bioeng 2009; 2: 57-65.

54. Walshaw J, Woolfson DN. Socket: a program for identifying and analysing coiled-coil motifs within protein structures. J Mol Biol 2001; 307: 1427-50.

55. Testa OD, Moutevelis E, Woolfson DN. CC+: a relational database of coiled-coil structures. Nucleic Acids Res 2009; 37: D315-22.

56. Szappanos B, Süveges D, Nyitray L, Perczel A, Gaspari Z. Folded-unfolded cross-predictions and protein evolution: the case study of coiled-coils. FEBS Lett 2010; 584: 1623-7.

57. Gruber M, Soding J, Lupas AN. Comparative analysis of coiled-coil prediction methods. J Struct Biol 2006; 155: 140-5.

58. Lupas A, Van Dyke M, Stock J. Predicting coiled coils from protein sequences. Science 1991; 252: 1162-4.

59. Gruber M, Soding J, Lupas AN. REPPER-repeats and their periodicities in fibrous proteins. Nucleic Acids Res 2005; 33 : W239-43.

60. Berger B, Wilson DB, Wolf E, Tonchev T, Milla M, Kim PS. Predicting coiled coils by use of pairwise residue correlations. Proc Natl Acad Sci USA 1995; 92: 8259-63.

61. McDonnell AV, Jiang T, Keating AE, Berger B. Paircoil2: improved prediction of coiled coils from sequence. Bioinformatics 2006; 22: 356-8.

62. Delorenzi M, Speed T. An HMM model for coiled-coil domains and a comparison with PSSM-based predictions. Bioinformatics 2002; 18: 617-25.

63. Wolf E, Kim PS, Berger B. MultiCoil: a program for predicting two- and three-stranded coiled coils. Protein Sci 1997; 6: 1179-89.

64. Bartoli L, Fariselli P, Krogh A, Casadio R. CCHMM_PROF: a HMM-based coiled-coil predictor with evolutionary information. Bioinformatics 2009; 25: 2757-63.

65. Rackham OJ, Madera M, Armstrong CT, Vincent TL, Woolfson DN, Gough J. The evolution and structure prediction of coiled coils across all genomes. J Mol Biol 2010; 403: 480-93.

66. Morii H, Takenawa T, Arisaka F, Shimizu T. Identification of kinesin neck region as a stable $\alpha$-helical coiled coil and its thermodynamic characterization. Biochemistry 1997; 36: 1933-42.

67. Tanizawa H, Ghimire GD, Mitaku S. A high performance prediction system of coiled coil domains containing heptad breaks: SOSUIcoil. Chem-Bio Info J 2008; 8: 96-111.

68. Lupas A. Predicting coiled-coil regions in proteins. Curr Opin Struct Biol 1997; 7: 388-93.

69. Fong JH, Keating AE, Singh M. Predicting specificity in bZIP coiled-coil protein interactions. Genome Biol 2004; 5: R11.

70. Rose A, Manikantan S, Schraegle SJ, Maloy MA, Stahlberg EA, Meier I. Genome-wide identification of arabidopsis coiled-coil proteins and establishment of the ARABI-COIL database. Plant Physiol 2004; 134: 927-39.

71. Walshaw J, Gillespie MD, Kelemen GH. A novel coiled-coil repeat variant in a class of bacterial cytoskeletal proteins. $\mathrm{J}$ Struct Biol 2010; 170: 202-15.

72. Odgren PR, Harvie LW Jr, Fey EG. Phylogenetic occurrence of coiled coil proteins: implications for tissue structure in metazoa via a coiled coil tissue matrix. Proteins 1996; 24: 467-84.

73. Rose A, Schraegle SJ, Stahlberg EA, Meier I. Coiled-coil protein composition of 22 proteomes - differences and common themes in subcellular infrastructure and traffic control. BMC Evol Biol 2005; 5: 66.

74. Guo Y, Kammerer RA, Engel J. The unusually stable coiledcoil domain of COMP exhibits cold and heat denaturation in 4-6 M guanidinium chloride. Biophys Chem 2000; 85: $179-86$.

75. Kim Y, Heuser JE, Waterman CM, Cleveland DW. CENP-E combines a slow, processive motor and a flexible coiled coil to produce an essential motile kinetochore tether. J Cell Biol 2008; 181: 411-9.

76. Ohtaki A, Kida H, Miyata Y, Ide N, Yonezawa A, Arakawa T, Iizuka R, Noguchi K, Kita A, Odaka M, Miki K, Yohda M. Structure and molecular dynamics simulation of archaeal prefoldin: the molecular mechanism for binding and recognition of nonnative substrate proteins. J Mol Biol 2008; 376: 1130-41.

77. Palle K, Pattarello L, van der Merwe M, Losasso C, Benedetti P, Bjornsti MA. Disulfide cross-links reveal conserved features of DNA topoisomerase I architecture and a role for the $\mathrm{N}$ terminus in clamp closure. J Biol Chem 2008; 283: 27767-75.

78. Perry JJ, Asaithamby A, Barnebey A, Kiamanesch F, Chen DJ, Han S, Tainer JA, Yannone SM. Identification of a coiled coil in Werner syndrome protein that facilitates multimerization and promotes exonuclease processivity. J Biol Chem 2010; 285: 25699-707.

79. Schonichen A, Bigalke JM, Urbanke C, Grzesiek S, Dames SA, Geyer M. A flexible bipartite coiled coil structure is required for the interaction of Hexim1 with the P-TEFB subunit cyclin T1. Biochemistry 2010; 49: 3083-91.

80. Tuteja D, Rafizadeh S, Timofeyev V, Wang S, Zhang Z, Li N, Mateo RK, Singapuri A, Young JN, Knowlton AA, Chiamvimonvat $\mathrm{N}$. Cardiac small conductance $\mathrm{Ca}^{2+}$-activated $\mathrm{K}^{+}$ channel subunits form heteromultimers via the coiled-coil domains in the C-termini of the channels. Circ Res 2010; 107: 851-9.

81. Barbar E. Dynein light chain LC8 is a dimerization hub essential in diverse protein networks. Biochemistry 2008; 47: $503-8$. 
82. Hodi Z, Rapali P, Radnai L, Molnar T, Szenes A, Kardos J, Buday L, Stafford W, Nyitray L. The LC8 family of dynein light chains: multifunctional chaperon-like proteins. FEBS J 2007; 274: 106.

83. Strauss HM, Keller S. Pharmacological interference with protein-protein interactions mediated by coiled-coil motifs. Handb Exp Pharmacol 2008; 186: 461-82.

84. McFarlane AA, Orriss GL, Stetefeld J. The use of coiled-coil proteins in drug delivery systems. Eur J Pharmacol 2009; 625: $101-7$.

85. Mittl PR, Deillon C, Sargent D, Liu N, Klauser S, Thomas RM, Gutte B, Grutter MG. The retro-GCN4 leucine zipper sequence forms a stable three-dimensional structure. Proc Natl Acad Sci USA 2000; 97: 2562-6.

86. Lovejoy B, Choe S, Cascio D, McRorie DK, DeGrado WF, Eisenberg D. Crystal structure of a synthetic triple-stranded $\alpha$-helical bundle. Science 1993; 259: 1288-93.

87. Root BC, Pellegrino LD, Crawford ED, Kokona B, Fairman R. Design of a heterotetrameric coiled coil. Protein Sci 2009; 18: $329-36$.

88. Grigoryan G, Keating AE. Structural specificity in coiled-coil interactions. Curr Opin Struct Biol 2008; 18: 477-83.

89. Walshaw J, Woolfson DN. Open-and-shut cases in coiled-coil assembly: $\alpha$-sheets and $\alpha$-cylinders. Protein Sci 2001; 10: $668-73$.

90. Tompa P. Structure and function of intrinsically disordered proteins. CRC Press: Boca Raton, FL, 2009.

91. Iakoucheva LM, Brown CJ, Lawson JD, Obradovic Z, Dunker AK. Intrinsic disorder in cell-signaling and cancer-associated proteins. J Mol Biol 2002; 323: 573-84.

92. Sivaramakrishnan S, Spink BJ, Sim AY, Doniach S, Spudich JA. Dynamic charge interactions create surprising rigidity in the ER/K $\alpha$-helical protein motif. Proc Natl Acad Sci USA 2008; 105: 13356-61.

93. Süveges D, Gáspári Z, Tóth G, Nyitray L. Charged single $\alpha-$ helix: a versatile protein structural motif. Proteins 2009; 74 : 905-16.

94. Dobson CM. Protein misfolding, evolution and disease. Trends Biochem Sci 1999; 24: 329-32.
95. Perczel A, Hudaky P, Palfi VK. Dead-end street of protein folding: thermodynamic rationale of amyloid fibril formation. J Am Chem Soc 2007; 129: 14959-65.

96. Choma C, Gratkowski H, Lear JD, DeGrado WF. Asparaginemediated self-association of a model transmembrane helix. Nat Struct Biol 2000; 7: 161-6.

97. Zhou FX, Cocco MJ, Russ PR, Brunger AT, Engelman DM. Interhelical hydrogen bonding drives strong interactions in membrane proteins. Nat Struct Biol 2000; 7: 154-60.

98. Ortlund EA, Bridgham JT, Redinbo MR, Thornton JW. Crystal structure of an ancient protein: evolution by conformational epistasis. Science 2007; 317: 1544-8.

99. Groves MR, Barford D. Topological characteristics of helical repeat proteins. Curr Opin Struct Biol 1999; 9: 383-9.

100. Odronitz F, Kollmar M. Drawing the tree of eukaryotic life based on the analysis of 2,269 manually annotated myosins from 328 species. Genome Biol 2007; 8: R196.

101. Richards TA, Cavalier-Smith T. Myosin domain evolution and the primary divergence of eukaryotes. Nature 2005; 436: 1113-8.

102. Peckham M, Knight PJ. When a predicted coiled coil is really a single $\alpha$-helix, in myosins and other proteins. Soft Matter 2009; 5: 2493-503.

103. Sato Y, Shirakawa R, Horiuchi H, Dohmae N, Fukai S, Nureki O. Asymmetric coiled-coil structure with guanine nucleotide exchange activity. Structure 2007; 15: 245-52.

104. Pervushin K, Vamvaca K, Vogeli B, Hilvert D. Structure and dynamics of a molten globular enzyme. Nat Struct Mol Biol 2007; 14: 1202-6.

105. Kraulis PJ. MOLSCRIPT: a program to produce both detailed and schematic plots of protein structures. J Appl Cryst 1991; 24: $946-50$

106. Dosztányi Z, Csizmók V, Tompa P, Simon I. IUPRed: web server for the prediction of intrinsically unstructured regions of proteins based on estimated energy content. Bioinformatics 2005; 21: 3433-4.

Received October 19, 2010; accepted March 1, 2011 